\title{
Volume matching condition to establish the enhanced piezoelectricity in ternary $(\mathrm{Sc}, \mathrm{Y})_{0.5}$ $(\mathrm{Al}, \mathrm{Ga}, \mathrm{In})_{0.5} \mathrm{~N}$ alloys
}

Christopher Tholander, Igor Abrikosov, Lars Hultman and Ferenc Tasnadi

\section{Linköping University Post Print}

\section{Tweet}

N.B.: When citing this work, cite the original article.

Original Publication:

Christopher Tholander, Igor Abrikosov, Lars Hultman and Ferenc Tasnadi, Volume matching condition to establish the enhanced piezoelectricity in ternary $(\mathrm{Sc}, \mathrm{Y}){ }_{0.5}(\mathrm{Al}, \mathrm{Ga}, \mathrm{In}){ }_{0.5} \mathrm{~N}$ alloys, 2013, Physical Review B. Condensed Matter and Materials Physics, (87), 9.

http://dx.doi.org/10.1103/PhysRevB.87.094107

Licencee: American Physical Society

http://www.aps.org/

Postprint available at: Linköping University Electronic Press http://urn.kb.se/resolve?urn=urn:nbn:se:liu:diva-91013 


\title{
Volume matching condition to establish the enhanced piezoelectricity in ternary $(\mathrm{Sc}, \mathrm{Y})_{0.5}(\mathrm{Al}, \mathrm{Ga}, \mathrm{In})_{0.5} \mathrm{~N}$ alloys
}

\author{
Christopher Tholander, ${ }^{*}$ Igor A. Abrikosov, Lars Hultman, and Ferenc Tasnádi \\ Department of Physics, Chemistry and Biology (IFM), Linköping University, SE-581 83 Linköping, Sweden
}

(Received 22 December 2012; revised manuscript received 4 March 2013; published 12 March 2013)

\begin{abstract}
Recently, ScAIN alloys attracted attention for their giant piezoelectric moduli. In this study the piezoelectric response of the wurtzite group-III nitrides $\mathrm{AlN}, \mathrm{GaN}$, and $\mathrm{InN}$ mixed with $50 \mathrm{~mol} \%$ of $\mathrm{ScN}$ or $\mathrm{YN}$ is investigated using $a b$ initio calculations. We confirm that the energy flattening phenomenon gives rise to the simultaneous appearance of elastic softening and local structural instability, and explains the enhanced piezoelectricity of the alloys. Furthermore, we present a volume matching condition for an efficient search of new piezoelectric materials. It states that alloys in which the parent components show close volume matching exhibit a flatter potential-energy landscape and higher increase of piezoelectric moduli. We suggest YInN, beyond ScAlN, as a promising material for piezoelectric energy harvesting with its enhanced $\approx 400 \%$ piezoelectric moduli.
\end{abstract}

DOI: 10.1103/PhysRevB.87.094107

PACS number(s): 77.65.-j, 61.66.Dk, 71.15.Mb

\section{INTRODUCTION}

Energy harvesting, saving, or reducing the energy consumption of microelectronic devices is a multidisciplinary challenge from materials science to device fabrication. ${ }^{1,2}$ For example, piezoelectric nanowires support the development of self-powered nanosystems. ${ }^{3}$ Piezoelectric energy harvesting is based on the piezoelectric effect, ${ }^{4}$ where vibrational energy is converted into electrical by a piezoelectric material. The operational efficiency of a vibrational piezoelectric cantilever integrated into a microelectromechanical system (MEMS) has been investigated both experimentally ${ }^{5}$ and theoretically. ${ }^{6}$ The characteristic of a piezoelectric power MEMS is determined by material factors, such as the piezoelectric coefficients $\left(e_{i j}\right)$, dielectric constants $(\varepsilon)$, electromechanical coupling $(k)$, and mechanical quality factor $(Q)$ of the applied piezoelectric material. The power output of a MEMS is proportional to the piezoelectric response, but decreases with higher $\varepsilon$.

Ferroelectric single crystal and polycrystalline lead zirconate titanate (PZT) based materials have high piezoelectric response and electromechanical coupling factor, which makes them suitable for MEMS. However, their also high dielectric constant counterbalances these advantages in terms of the power output. ${ }^{7}$ Accordingly, materials with power outputs similar to that of PZT recently received more scientific and technological interest. Aluminum nitride (AlN) based thin films offer promising alternatives due to AlN's comparable power output coupled with an extremely high $Q$ value. $^{8}$ Furthermore, its piezoelectric response has a high temperature stability, up to $1150^{\circ} \mathrm{C}$. The major drawback of wurtzite AlN is that it exhibits a low piezoelectric moduli $\left(d_{i j}\right)$ around $5.5 \mathrm{pCN}^{-1}$ in comparison to $410 \mathrm{pCN}^{-1}$ of PZT. ${ }^{9}$

$\mathrm{Sc}_{x} \mathrm{Al}_{1-x} \mathrm{~N}$ alloys were found to have a giant $\approx 600 \%$ increase of the piezoelectric moduli $d_{33}$ at around $x=$ 0.5 , in reference to pure wurtzite AlN. ${ }^{9,10}$ The microscopic physical origin of this anomalous increase was explained by the flattening of the potential energy landscape, which results simultaneously in elastic softening and local structural instability. ${ }^{10}$ This generic phenomena was later validated also for ferroelectric materials, in relaxor oxides, in the context of morphotropic phase boundaries (MPB).$^{11}$ Thin-film ScAlN has been grown and its electromechanical and dielectric prop- erties were investigated experimentally. ${ }^{12}$ The huge increase of the piezoelectric response, with only a moderate increase of the dielectric constant was observed. Accordingly, groupIII-nitride-based alloys should be considered as candidates for energy harvesting and MEMS. Recently, YAIN alloys have been investigated experimentally and theoretically. ${ }^{13}$

Mixing different materials in various amounts appoints a high dimensional parameter space that one can explore in a high-throughput manner. A computational realization of this technique has been applied successfully in searching for highperformance piezoelectric perovskites ${ }^{14}$ However, a drawback of the approach is its huge computational demand. Yet, it can be resolved by reducing the combinatorial freedoms by applying simple rules of thumb to filter the candidates before investigation.

Here, we calculate and compare the piezoelectric response of several group-III-nitride-based alloys. We study the energy flattening phenomena discovered for ScAlN and compare the potential-energy landscapes of $\mathrm{Sc}_{0.5} \mathrm{Al}_{0.5} \mathrm{~N}, \mathrm{Sc}_{0.5} \mathrm{Ga}_{0.5} \mathrm{~N}$, $\mathrm{Sc}_{0.5} \mathrm{In}_{0.5} \mathrm{~N}, \mathrm{Y}_{0.5} \mathrm{Al}_{0.5} \mathrm{~N}, \mathrm{Y}_{0.5} \mathrm{Ga}_{0.5} \mathrm{~N}$, and $\mathrm{Y}_{0.5} \mathrm{In}_{0.5} \mathrm{~N}$ alloys. Through the comparison we confirm the presence of the energy flattening and find the volume matching of the parent binary components to be an indicator of the enhancement of the piezoelectric moduli. Furthermore, we suggest YInN as a promising candidate for piezoelectric energy harvesting.

\section{METHOD}

The special quasirandom structure (SQS) $)^{15,16}$ method was used to model the substitutional disorder in the alloys. The SQS supercell with a 50/50 composition was constructed and used for all the alloys. It has been generated by optimizing the Warren-Cowley short-range order parameters up to the seventh coordination shell. An appropriate solid solution model was obtained with the supercell size of $(4 \times 2 \times 4)$ that consists of 128 atomic sites. This structural model was successfully used previously in explaining the microscopic origin of the giant piezoelectric response in ScAIN. ${ }^{10}$ The SQS structure can be found in the Supplemental Material. ${ }^{17}$

The calculations were performed by using density-functional theory within the generalized gradient 
approximation (Perdew-Burke-Ernzerhof GGA). ${ }^{18}$ Though there are concerns about the performance of GGA for ferroelectric oxides, ${ }^{19,20}$ polarization properties are moderately sensitive to the exchange-correlation functional; also GGA slightly outperforms LDA when considering the III-V nitrides, which are the objects of this study. ${ }^{21}$ We used the QUANTUM-ESPRESSO (QE) package ${ }^{22}$ with Vanderbilt ultrasoft pseudopotentials. ${ }^{23}$ The proper piezoelectric constants $e_{33}$ (Ref. 24) were calculated with the modern Berry-phase approach as implemented in QE. The elastic constants $C_{33}$ were derived from total energy calculations. A finite difference technique with $\pm 1,2 \%$ lattice distortion was applied to obtain the derivatives of both the total energy and the Berry phase with respect to the strain component $\epsilon_{33}$.

The plane-wave cutoff energy together with the MonkhorstPack sampling of the Brillouin zone were converged until a sufficient convergence was achieved. We reproduced the bulk elastic and piezoelectric constants of group-III nitrides in good agreement with literature data. ${ }^{25} \mathrm{~A}$ full structural relaxation of all the SQS supercells was performed. As the purpose was to establish the topology of the potential-energy landscapes, the $c / a$ ratio and cell volume were treated separately. In the case of YGaN we used instead the variable cell shape ionic relaxation method in QE with a $0.05 \mathrm{GPa}$ convergence threshold to find the energy minima.

The potential-energy flattening in the alloys is connected to the structures of the parent binary materials. Group-III nitrides ( $\mathrm{AlN}, \mathrm{GaN}$, and $\mathrm{InN}$ ) form the wurtzite (B4) structure in their ground state, while $\mathrm{ScN}$ and $\mathrm{YN}$ form a layered hexagonal structure. The layered hexagonal phase differs from the wurtzite structure only in the internal parameter $u$, the ratio of the shift between the metal and nitrogen sublattices. The layered hexagonal phase is described with $u=0.5$, while for the wurtzite phase $u \neq 0.5$. In ScAlN, the inherited wurtzite and hexagonal phases are energetically close to each other, which is what flattens the potential-energy landscape along the $c$ axis. ${ }^{10}$ This topological change explains the simultaneous occurrence of the softening of $C_{33}$ that results in the giant enhancement of the piezoelectric moduli $d_{33}$.

Potential-energy landscapes of ScGaN, ScInN, YAlN, and YInN were derived by an interpolation of energy points obtained on a $(5 \times 5)$ grid in the (volume, $c / a)$ space together with some additional points around the minima. The potentialenergy cross sections of the surfaces were calculated by finding the minimum energy values for each $c / a$ in the interpolated surface. In the same manner as the energy, a landscape of the internal parameter $u$ was created from the average $u$ value in the relaxed supercells. Then, the values along the energy cross-section path were extracted. The wurtzite local environment (a tetrahedron) of each metal atom was investigated by the superposition of all the tetrahedra in the supercell. Since one finds two orientations of the tetrahedra in the supercell, the local environment of each atom is depicted as a metal atom in the center with six legs, where only $\mathrm{N}$ atoms sit.

\section{RESULTS AND DISCUSSION}

Figure 1 shows the calculated potential-energy landscapes of $\mathrm{Y}_{0.5} \mathrm{Al}_{0.5} \mathrm{~N}, \mathrm{Sc}_{0.5} \mathrm{In}_{0.5} \mathrm{~N}$, and $\mathrm{Sc}_{0.5} \mathrm{Ga}_{0.5} \mathrm{~N}$. It reveals three

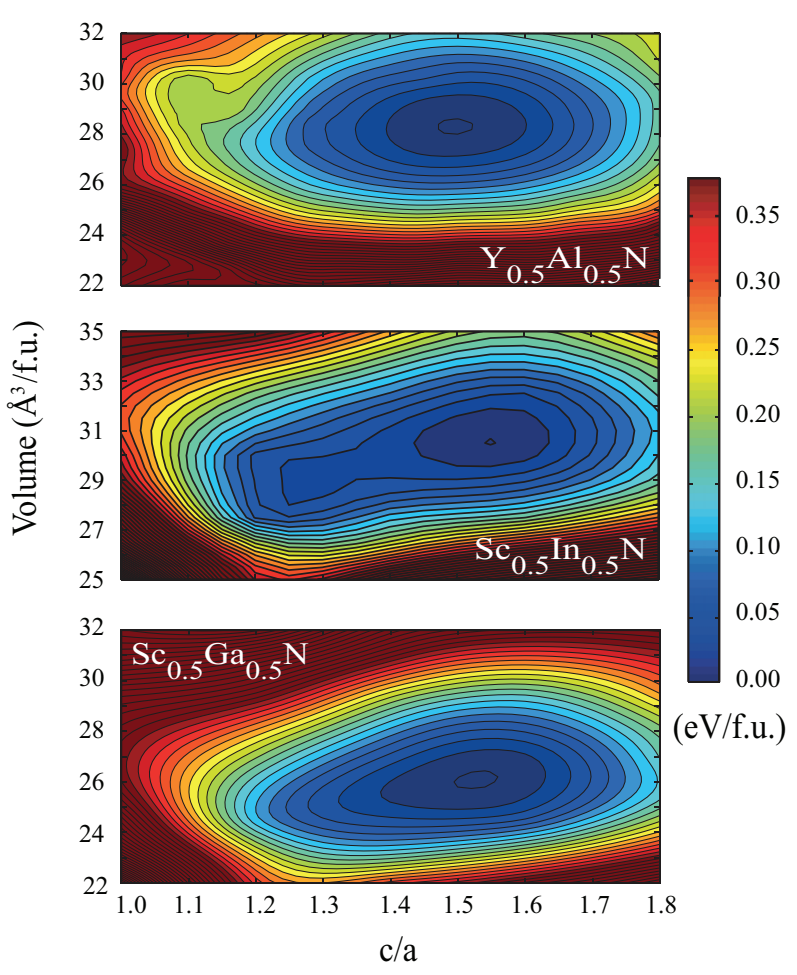

FIG. 1. (Color online) Potential-energy landscapes of $\mathrm{Y}_{0.5} \mathrm{Al}_{0.5} \mathrm{~N}$, $\mathrm{Sc}_{0.5} \operatorname{In}_{0.5} \mathrm{~N}$, and $\mathrm{Sc}_{0.5} \mathrm{Ga}_{0.5} \mathrm{~N}$. The elongation of the energy minima is a sign of an increased $e_{33}$ and a decreased $C_{33}$ that will improve $d_{33}$. The characteristic shape of the $\mathrm{Sc}_{0.5} \mathrm{Ga}_{0.5} \mathrm{~N}$ energy landscape results in the strongest increase of $d_{33}$.

scenarios in this material class. First, in the case of $\mathrm{Y}_{0.5} \mathrm{Al}_{0.5} \mathrm{~N}$ the hexagonal and wurtzite energy minima are energetically very far from each other. Second, for $\mathrm{Sc}_{0.5} \mathrm{In}_{0.5} \mathrm{~N}$ one sees that the two phases are already very close in energy, though the potential-energy landscape shows a double-well structure. The layered hexagonal structure energy minimum is at $c / a \approx$ 1.3 , while the wurtzite structure minimum is located at $c / a \approx$ 1.6. Finally, $\mathrm{Sc}_{0.5} \mathrm{Ga}_{0.5} \mathrm{~N}$ likewise to $\mathrm{Sc}_{0.5} \mathrm{Al}_{0.5} \mathrm{~N}$ (see Ref. 10) shows a flat, elongated potential-energy landscape between the two phases.

Further characteristics can be extracted with the help of the potential-energy cross sections. Figure 2 shows the cross sections of $\mathrm{Y}_{0.5} \mathrm{Al}_{0.5} \mathrm{~N}, \mathrm{Sc}_{0.5} \mathrm{In}_{0.5} \mathrm{~N}$, and $\mathrm{Sc}_{0.5} \mathrm{Ga}_{0.5} \mathrm{~N}$ together with the corresponding cross sections of the parent binary group-III nitrides. It also shows the extracted average wurtzite internal parameter $u$. One sees that the change of $u$ from the value 0.5 coincides with the positions of the layered hexagonal structure energy minima. However, small deviations from $u=0.5$ exist even within the stability field of that structure. This comes from the averaging of $u$ over the entire supercell, which contains local differences in $u$. Furthermore, in all ternary alloys the wurtzite structure energy minimum is shifted significantly to a lower $c / a$ value compared to the parent group-III nitrides. The same effect has been observed for $\mathrm{Sc}_{0.5} \mathrm{Al}_{0.5} \mathrm{~N} .{ }^{10}$ In $\mathrm{Y}_{0.5} \mathrm{Al}_{0.5} \mathrm{~N}$, both the hexagonal and wurtzite structure energy minima are shifted rather as a coupled pair. The decrease of the $c / a$ ratio can be explained by the larger increase of the lattice parameter $a$ compared to $c$ caused by enforcing the layered hexagonal phase, flattening the internal 

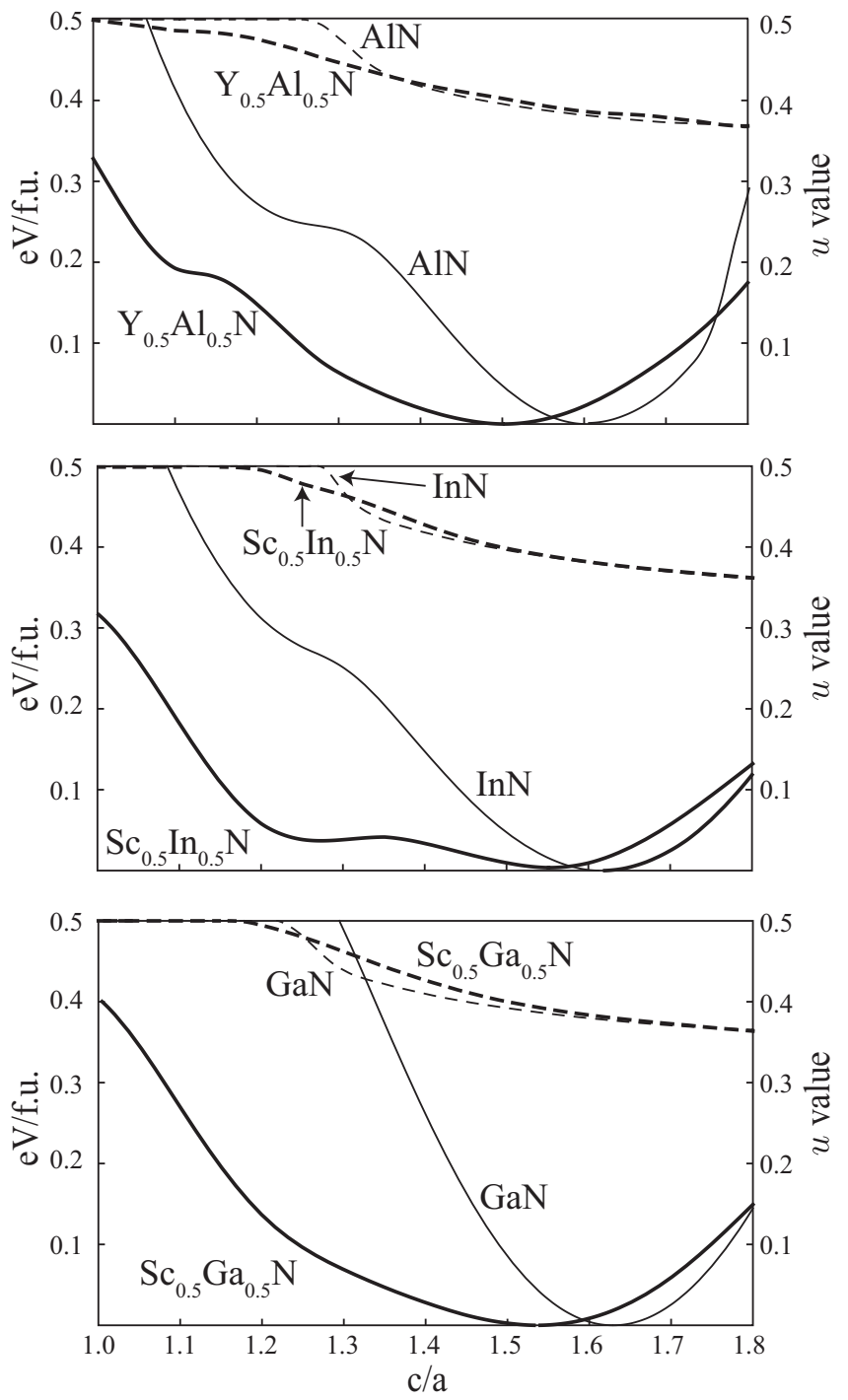

FIG. 2. Potential-energy landscape cross sections for $\mathrm{Y}_{0.5} \mathrm{Al}_{0.5} \mathrm{~N}$, $\mathrm{Sc}_{0.5} \mathrm{In}_{0.5} \mathrm{~N}$, and $\mathrm{Sc}_{0.5} \mathrm{Ga}_{0.5} \mathrm{~N}$ (thick solid lines) and the corresponding $u$ values (thick dashed lines). Thin lines show the same quantities for the parent binary wurtzite group-III nitrides.

structure. The small decrease of $c / a$ in the case of $\operatorname{Sc}_{0.5} \operatorname{In}_{0.5} \mathrm{~N}$ explains the double-well structure of the energy landscape and prevents the simultaneous appearance of elastic softening and

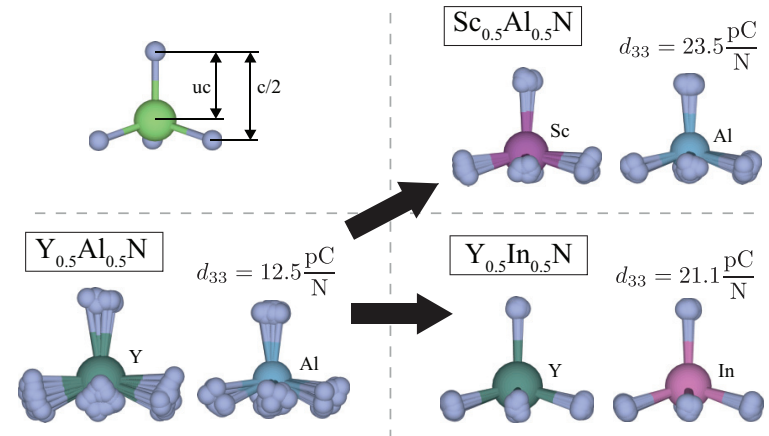

FIG. 3. (Color online) The local tetrahedral environments around the metal atoms in $\mathrm{Y}_{0.5} \mathrm{Al}_{0.5} \mathrm{~N}, \mathrm{Sc}_{0.5} \mathrm{Al}_{0.5} \mathrm{~N}$, and $\mathrm{Y}_{0.5} \mathrm{In}_{0.5} \mathrm{~N}$ along with a model showing the $u$ parameter.

local structural instability. We emphasize that the decrease of $c / a$, compared to the parent group-III nitride, is very beneficial for the enhancement of the piezoelectric response since it favors the smooth flattening of the energy landscape between the wurtzite and layered hexagonal phase energy minima.

We calculated the elastic $C_{33}$ and piezoelectric constants $e_{33}$ of both the parent binaries and alloys. The values of $d_{33}$ are approximated with the relation $d_{33} \approx e_{33} / C_{33}$, and presented in Table I. A large increase of $d_{33}$ requires high $e_{33} / e_{33}^{\text {parent }}$ and low $C_{33} / C_{33}^{\text {parent }}$ values, simultaneously. $\mathrm{Sc}_{0.5} \mathrm{Ga}_{0.5} \mathrm{~N}$ gives an excellent increase of $d_{33}$ from 1.86 to $16.90 \mathrm{pC} / \mathrm{N}$ in correspondence with the expectations from Fig. 1. Remarkably, $\mathrm{Y}_{0.5} \mathrm{In}_{0.5} \mathrm{~N}$ and $\mathrm{Sc}_{0.5} \mathrm{Al}_{0.5} \mathrm{~N}$ exhibit very similar enhancements of $d_{33}$. Table I underlines the three characteristic scenarios shown in Fig. 1. The $d_{33}$ values express that mixing GaN with $\mathrm{ScN}$ enhances the piezoelectricity more than in the other two combinations. Furthermore, $\mathrm{Y}_{0.5} \mathrm{Al}_{0.5} \mathrm{~N}$ clearly outperforms $\mathrm{Sc}_{0.5} \operatorname{In}_{0.5} \mathrm{~N}$. In situations where the layered hexagonal and wurtzite structures are separated from each other, either energetically or geometrically (see $\mathrm{Y}_{0.5} \mathrm{Al}_{0.5} \mathrm{~N}$ and $\mathrm{Sc}_{0.5} \mathrm{In}_{0.5} \mathrm{~N}$ ), one obtains a low increase of the piezoelectric response $d_{33}$. However, if the potential-energy landscape topology is flat and elongated along the hexagonal $c$ axis $\left(\mathrm{Sc}_{0.5} \mathrm{Ga}_{0.5} \mathrm{~N}\right)$, a giant increase of the piezoelectric response is obtained. In accordance, we confirm the previously introduced energy flattening argument discovered in ScAlN. ${ }^{10}$ Table I also reveals that the increase of $d_{33}$ is larger when alloying AlN or GaN with $\mathrm{ScN}$ rather than $\mathrm{YN}$. However, for $\mathrm{InN}$, it is more favorable to

TABLE I. Piezoelectric constants for parent binaries and 50/50 compositions with $\mathrm{YN}$ and $\mathrm{ScN}$. $e_{33}$ given in $\mathrm{C} / \mathrm{m}^{2}, C_{33}$ in $\mathrm{GPa}$, and $d_{33} \approx e_{33} / C_{33}$ in $\mathrm{pC} / \mathrm{N}$.

\begin{tabular}{|c|c|c|c|c|c|c|}
\hline Material & $e_{33}$ & $e_{33} / e_{33}^{\text {parent }}$ & $C_{33}$ & $C_{33} / C_{33}^{\text {parent }}$ & $d_{33}$ & $d_{33}$ increase $(\%)$ \\
\hline $\mathrm{InN}$ & 1.09 & & 211.4 & & 5.18 & \\
\hline $\mathrm{GaN}$ & 0.66 & & 357.7 & & 1.86 & \\
\hline AlN & 1.38 & & 342.0 & & 4.03 & \\
\hline $\mathrm{Y}_{0.5} \operatorname{In}_{0.5} \mathrm{~N}$ & 2.26 & 2.07 & 107.1 & 0.51 & 21.10 & 408 \\
\hline $\mathrm{Sc}_{0.5} \mathrm{In}_{0.5} \mathrm{~N}$ & 1.47 & 1.34 & 153.1 & 0.72 & 9.60 & 186 \\
\hline $\mathrm{Y}_{0.5} \mathrm{Ga}_{0.5} \mathrm{~N}$ & 1.85 & 2.78 & 191.0 & 0.53 & 9.67 & 521 \\
\hline $\mathrm{Sc}_{0.5} \mathrm{Ga}_{0.5} \mathrm{~N}$ & 2.30 & 3.46 & 135.9 & 0.38 & 16.90 & 911 \\
\hline $\mathrm{Y}_{0.5} \mathrm{Al}_{0.5} \mathrm{~N}$ & 2.15 & 1.56 & 171.4 & 0.50 & 12.54 & 311 \\
\hline $\mathrm{Sc}_{0.5} \mathrm{Al}_{0.5} \mathrm{~N}$ & 3.05 & 2.22 & 130.0 & 0.38 & 23.46 & 583 \\
\hline
\end{tabular}




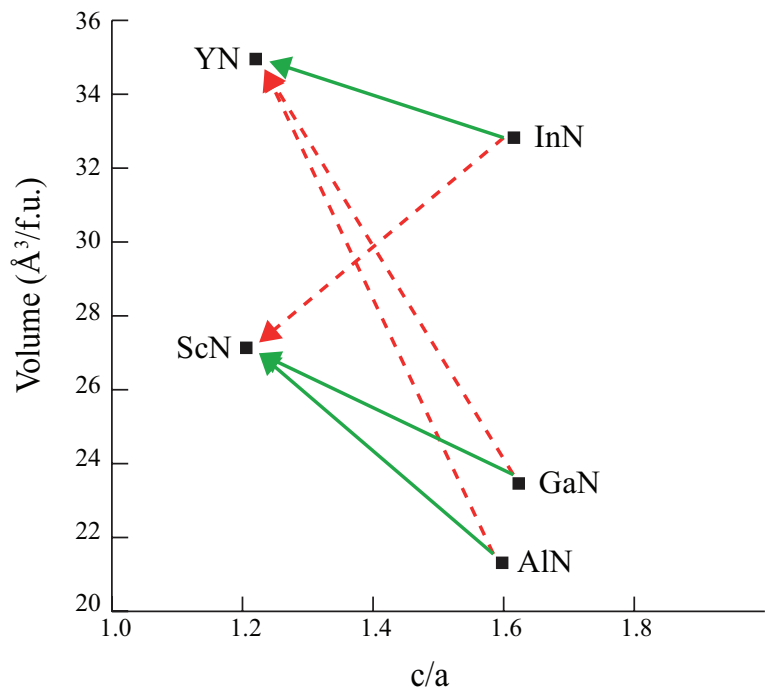

FIG. 4. (Color online) Volume and $c / a$ ratio of the binary compounds considered in this study. Solid lines show the alloy combinations with the most improved $d_{33}$ and dashed lines show combinations that will only produce a moderate increase.

use $\mathrm{YN}$ than $\mathrm{ScN}$. We note that $\mathrm{YInN}$ shows the second best increase of $d_{33}$.

Figure 3 shows all the 32 local tetrahedral environments in the alloys $\mathrm{Y}_{0.5} \mathrm{Al}_{0.5} \mathrm{~N}, \mathrm{Y}_{0.5} \operatorname{In}_{0.5} \mathrm{~N}$, and $\mathrm{Sc}_{0.5} \mathrm{Al}_{0.5} \mathrm{~N}$. The dispersions of the legs of these tetrahedra differ noticeably among the alloys. The size of the distortion of the local tetrahedral environment is dependent on the doping. A larger volume difference between the parent materials causes a larger dispersion of the legs. The largely distorted structure is also less sensitive to changes in the internal polarization in response to strain. Thus, the small size of the leg's dispersion is an additional marker for the favored alloying components, for example, mixing AlN with $\mathrm{ScN}$ instead of $\mathrm{YN}$. This is indicated with the big bold arrows in Fig. 3.

In alloys the volume difference between the components gives the major contribution to the local structural distortions. Thus, one expects a significant impact of the volume difference on the enhancement of the piezoelectric response. Figure 4 shows the volumes of the parent binaries $\mathrm{YN}, \mathrm{ScN}, \mathrm{InN}, \mathrm{GaN}$, and AlN.

The solid lines in Fig. 4 connect the binary pairs from Table I, which show a large increase of $d_{33}$, while the dashed lines designate the pairs giving lower enhancement. The figure expresses that if the volume difference is large, like in YAlN and YGaN, the energy landscape is not flat enough to support an anomalous increase of the piezoelectricity. The double-well-like scenario of $\mathrm{Sc}_{0.5} \operatorname{In}_{0.5} \mathrm{~N}$ can also be explained by the volume difference. Since the layered hexagonal $\mathrm{ScN}$ has a significantly smaller volume than the wurtzite $\mathrm{InN}$, the structural disturbance results in a small reduction of $(c / a)$ and prevents an elongated potential-energy landscape.

\section{CONCLUSIONS}

We compared the enhancement of the piezoelectric response in group-III nitrides mixed with $\mathrm{ScN}$ or $\mathrm{YN}$. The previously introduced energy flattening phenomena explaining the anomalous increase of the materials piezoelectric response is investigated and three major characteristic topologies were determined. (1) For $\mathrm{Y}_{0.5} \mathrm{Al}_{0.5} \mathrm{~N}$, the potential-energy landscape did not show satisfactory flattening; (2) the landscape of $\mathrm{Sc}_{0.5} \operatorname{In}_{0.5} \mathrm{~N}$ attained flattening, though with a double-well-like structure; and (3) in $\mathrm{Sc}_{0.5} \mathrm{Ga}_{0.5} \mathrm{~N}$ we found excellent energy flattening similar to ScAlN. We calculated the elastic $\left(C_{33}\right)$ and piezoelectric $\left(e_{33}\right)$ constants and investigated the internal structural instability with the help of the local tetrahedra environments around the metal atoms, and found the simultaneous occurrence of the elastic softening and structural instability. Finally, we established a simple volume matching argument to select alloying components in a search for a giant increase of the piezoelectric response. Using this argument we explained the observed topological characteristics of the potential-energy landscapes. Accordingly, we determined a rule of thumb to select the alloying material for the giant enhancement of the piezoelectric moduli of group-III nitrides. This rule, in correspondence with the calculations, suggests $\mathrm{YInN}$ as a promising material with a giant $\approx 400 \%$ increase of the piezoelectric response $d_{33}$.

\section{ACKNOWLEDGMENTS}

Financial support was offered by the Swedish Research Council Linneaus Environment LiLi-NFM, and a Knut and Alice Wallenberg Scholar LH Grant. I.A.A. and F.T. acknowledge support from the Swedish Research Council via Grant No. 621-2011-4426, and the Swedish Foundation for Strategic Research (SSF) programs SRL Grant No. 10-0026 and "Multifilms." The simulations were carried out using supercomputer resources provided by the Swedish National Infrastructure for Computing (SNIC) at the National Supercomputer Center (NSC).

\footnotetext{
*chtho@ifm.liu.se

${ }^{1}$ F. Cottone, H. Vocca, and L. Gammaitoni, Phys. Rev. Lett. 102, 080601 (2009).

${ }^{2}$ J. A. Paradiso and T. Starner, IEEE Pervasive Comput. 4, 18 (2005).

${ }^{3}$ Z. L. Wang, Adv. Funct. Mater. 18, 3553 (2008).

${ }^{4} \mathrm{R}$. Newnham, Properties of Materials (Oxford University Press, New York, 2005).

${ }^{5}$ P. Muralt, Integr. Ferroelectr. 17, 297 (1997).

${ }^{6}$ M. Renaud, K. Karakaya, T. Sterken, P. Fiorini, C. van Hoof, and

R. Puers, Sens. Actuators A 145-146, 380 (2008).
}

${ }^{7}$ K. Wasa, T. Matsushima, H. Adachi, I. Kanno, and H. Kotera, J. Microelectromech. Syst. 21, 451 (2012).

${ }^{8}$ S. Trolier-McKinstry, and P. Muralt, J. Electroceram. 12, 7 (2004).

${ }^{9}$ M. Akiyama, T. Kamohara, K. Kano, A. Teshigahara, Y. Takeuchi, and N. Kawahara, Adv. Mater. 21, 593 (2009).

${ }^{10}$ F. Tasnádi, B. Alling, C. Höglund, G. Wingqvist, J. Birch, L. Hultman, and I. A. Abrikosov, Phys. Rev. Lett. 104, 137601 (2010).

${ }^{11}$ D. Damjanovic, Appl. Phys. Lett. 97, 062906 (2010).

${ }^{12}$ G. Wingqvist, F. Tasnádi, A. Zukauskaite, J. Birch, H. Arwin, and L. Hultman, Appl. Phys. Lett. 97, 112902 (2010). 
${ }^{13}$ A. Zukauskaite, C. Tholander, J. Palisaitis, P. O. Å. Persson, V. Darakchieva, N. B. Sedrine, F. Tasnádi, B. Alling, J. Birch, and L. Hultman, J. Phys. D: Appl. Phys. 45, 422001 (2012).

${ }^{14}$ R. Armiento, B. Kozinsky, M. Fornari, and G. Ceder, Phys. Rev. B 84, 014103 (2011).

${ }^{15}$ A. Zunger, S. H. Wei, L. G. Ferreira, and J. E. Bernard, Phys. Rev. Lett. 65, 353 (1990).

${ }^{16}$ A. V. Ruban and I. A. Abrikosov, Rep. Prog. Phys. 71, 046501 (2008).

${ }^{17}$ See Supplemental Material at http://link.aps.org/supplemental/ 10.1103/PhysRevB.87.094107 for the details of SQS structure.

${ }^{18}$ J. P. Perdew, K. Burke, and M. Ernzerhof, Phys. Rev. Lett. 77, 3865 (1996).
${ }^{19}$ Z. Wu, R. E. Cohen, and D. J. Singh, Phys. Rev. B 70, 104112 (2004).

${ }^{20}$ D. I. Bilc, R. Orlando, R. Shaltaf, G. M. Rignanese, J. Iñiguez, and P. Ghosez, Phys. Rev. B 77, 165107 (2008).

${ }^{21}$ A. Zoroddu, F. Bernardini, P. Ruggerone, and V. Fiorentini, Phys. Rev. B 64, 045208 (2001).

${ }^{22}$ P. Giannozzi, S. Baroni, N. Bonini, M. Calandra, R. Car, C. Cavazzoni, D. Ceresoli, G. L. Chiarotti, M. Cococcioni, I. Dabo, J. Phys.: Condens. Matter 21, 395502 (2009).

${ }^{23}$ D. Vanderbilt, Phys. Rev. B 41, 7892 (1990).

${ }^{24}$ D. Vanderbilt, J. Phys. Chem. Solids 61, 147 (2000).

${ }^{25}$ F. Bernardini, V. Fiorentini, and D. Vanderbilt, Phys. Rev. B 56, R10024 (1997). 